\title{
FGFR1 Promotes Tumor Immune Evasion via YAP- Mediated PD-L1 Expression Upregulation in Lung Squamous Cell Carcinoma
}

\section{Min Lu}

Shanghai Jiao Tong University https://orcid.org/0000-0002-2499-1449

\section{Kaixuan Wang}

Shanghai Jiao Tong University Affiliated Chest Hospital

\section{Wenxiang Ji}

Shanghai Jiao Tong University Affiliated Chest Hospital

\section{Yongfeng Yu}

Shanghai Jiao Tong University Affiliated Chest Hospital

\section{Ziming Li}

Shanghai Jiao Tong University Affiliated Chest Hospital

\section{Weiliang Xia}

Shanghai Jiao Tong University

Shun Lu ( $\nabla$ shunlu@sjtu.edu.cn )

Shanghai Jiao Tong University https://orcid.org/0000-0001-8833-7262

\section{Research}

Keywords: FGFR1, PD-L1, driver mutation, tumor immune evasion, combination therapy

Posted Date: August 12th, 2021

DOl: https://doi.org/10.21203/rs.3.rs-770194/v1

License: (c) (i) This work is licensed under a Creative Commons Attribution 4.0 International License. Read Full License 
3 Min Lu ${ }^{1,2,3}$,zoe_lu_1995@hotmail.com

$4 \quad$ Kaixuan Wang ${ }^{1,3}$,wkx1xing@163.com

5 Wenxiang $\mathrm{Ji}^{1}$, jiwenxiange-mail@163.com

$6 \quad$ Yongfeng $\mathrm{Yu}^{1}$, yuyongfeng212@126.com

7 Ziming Li ${ }^{1}$, liziming1980@163.com

8 Weiliang Xia ${ }^{2}$,wlxia@sjtu.edu.cn

$9 \quad$ Shun Lu' ${ }^{1}$, shunlu@sjtu.edu.cn

$10{ }^{1}$ Shanghai Lung Cancer Center, Shanghai Chest Hospital, Shanghai Jiao Tong

11 University, West Huaihai Road 241, Shanghai, 20030, China.

$12{ }^{2}$ School of Biomedical Engineering and Med-X Research Institute, Shanghai Jiao

13 Tong University, Huashan Road 1954, Shanghai, 200030, China.

$14{ }^{3}$ These authors contributed equally to this work.

15

Corresponding authors: W. Xia, or S. Lu

W. Xia, PhD, State Key Laboratory of Oncogenes and Related Genes, School of Biomedical Engineering and Med-X Research Institute, Shanghai Jiao Tong University, Huashan Road 1954, Shanghai, 200240, China. Phone: +86-21-62933351; Fax: +86-21-62932302. E-mail: wlxia@sjtu.edu.cn

S. Lu, PhD MD, Shanghai Lung Cancer Center, Shanghai Chest Hospital, Shanghai Jiao Tong University, West Huaihai Road 241, Shanghai, 20030, China. Phone: +8621-62821990; Fax:+86-21-62933351. E-mail: shunlu@sjtu.edu.cn

Total Number of Figures: 7 figures and 2 supplementary figures. 


\section{Abstract}

2 Background: Variations in fibroblast growth factor receptor 1 (FGFR1), which occur 3 frequently, are common driver mutations of lung squamous cell carcinoma. Immune 4 checkpoint inhibitors targeting programmed death-1 (PD-1) and programmed death 5 ligand-1 (PD-L1) are powerful anticancer weapons. Activation of FGFR1 leads to protein (YAP), but whether and how FGFR1 regulates tumor immune evasion remain largely unclear.

Methods: H520 and HCC95 cells were treated with siRNA and plasmids to increase or decrease the expression of FGFR1, YAP and PD-L1, as assessed by molecular assays of protein and mRNA expression. The interaction between YAP and PD-L1 was verified by chromatin immunoprecipitation. After FGFR1 knockdown by shRNA, cancer cells were cocultured with Jurkat $\mathrm{T}$ cells, and then cell proliferation and activity were assessed. In C57BL/6 mice, the tumor immune microenvironment was analyzed by flow cytometry, immunofluorescence and immunohistochemistry after FGFR1 knockdown. The effect of the combination of FGFR1 knockdown and PD-1 blockade was explored both in vitro and in vivo.

Results: In H520 and HCC95 cells, FGFR1 upregulated PD-L1 expression via YAP, and YAP initiated the transcription of PD-L1 after binding to its promoter region. Both in vitro and in vivo, FGFR1 knockdown decreased tumor growth and reduced immune escape and reactivation of T cells. The combination of FGFR1 knockdown and PD-1 blockade synergistically exerted antitumor effects. In human LSQCC, the expression of fibroblast growth factor 2 (FGF2), the activator of FGFR1, was positively correlated with that of PD-L1 at the mRNA level.

Conclusions: The FGFR1/YAP/PD-L1 regulatory axis mediates tumor-associated immune suppression in lung squamous cell carcinoma, and FGFR1 knockdown reactivates $\mathrm{T}$ cells in the tumor microenvironment. Synergistic inhibition of both FGFR1 and PD-1/PD-L1 may be a possible treatment for lung cancer patients.

Keywords: FGFR1; PD-L1; driver mutation; tumor immune evasion; combination therapy. 


\section{Background}

Fibroblast growth factor receptor 1 (FGFR1) is a receptor tyrosine kinase and one of the four members of the FGFR family (1). Variations in FGFR1 exist in nearly 20\% of lung squamous cell carcinoma (LSQCC) patients (2-4), and they mediate proliferation, metastasis, epithelial-mesenchymal transition and stemness (5-7). However, the response rate to selective FGFR tyrosine kinase inhibitors (TKIs) and nonselective multitargeted FGFR TKIs is only $8 \%-12 \%$ in certain lung cancer patients (8-11). More evidence is needed to determine the unknown phenotypes of FGFR1 variations.

In the past decade, immune checkpoint inhibitors (ICIs), especially those targeting programmed death-1 (PD-1, or CD279) and programmed death ligand-1 (PD-L1, or CD274), have introduced the concept of immune therapy to lung cancer treatment (12). PD-L1 is abundantly expressed on cancer cells, macrophages, and dendritic cells (DCs). The interaction between PD-1 and PD-L1 causes T lymphocyte dysfunction, enabling cancer cells to escape immune surveillance (13-15). In several cancer types, pan-FGFR inhibitors positively remodel the immune profile within the tumor microenvironment (TME) (16-20), suggesting that FGFR1 may have an immunosuppressive effect.

Yes-associated protein (YAP) is the main downstream effector of the Hippo/LATS1 (also known as the Salvador-Warts-Hippo) transduction pathway (21). FGFR1 mediates the stemness of LSQCC cells by interacting with LATS1/YAP (6). When Hippo/LATS1 is inactivated, YAP is transported into the nucleus and forms a complex with transcriptional enhanced associate domain (TEAD) $(22,23)$. Activation of YAP/TAZ (another cotranscription factor like YAP) promotes PD-L1 transcription in lung adenocarcinoma, large cell lung cancer, breast cancer and melanoma cell lines, increasing the immune escape of cancer cells ${ }^{24-28}$. We wondered whether YAP also regulates the expression of PD-L1 in LSQCC.

In this study, we investigated the role of FGFR1 in the mechanisms of immune escape by LSQCC cells and, more specifically, on immune cells of the TME. Considering the limited response rate to targeted or immune therapy alone, the effect of the combination of FGFR1 inhibition and PD-1/PD-L1 blockade was explored both in vitro and in vivo. Our work uncovered a regulatory axis between FGFR1 and PD-L1 that may provide new insights into tumor immune evasion and clues for novel therapeutic interventions for LSQCC patients.

\section{Materials and methods \\ Cell culture and reagents}

H520, HCC95 and Jurkat T cells were purchased from ATCC and cultured using RPMI 1640 (HyClone, USA) with 10\% fetal bovine serum (Gibco, USA), 100 U/mL penicillin, and $100 \mu \mathrm{g} / \mathrm{mL}$ streptomycin (Gibco, USA). LLC cells were purchased from ATCC and cultured using DMEM (HyClone, USA) with 10\% fetal bovine serum (Gibco, USA), $100 \mathrm{U} / \mathrm{mL}$ penicillin, and $100 \mu \mathrm{g} / \mathrm{mL}$ streptomycin (Gibco, USA). FGF2 and IFN- $\gamma$ were purchased from PeproTech. AZD4547 and nivolumab were purchased from Selleck. AZD4547 was dissolved in DMSO, aliquoted, and stored as a $10 \mathrm{mM}$ stock solution at $-20^{\circ} \mathrm{C}$. Nivolumab was stored at $4{ }^{\circ} \mathrm{C}$. Verteporfin (Sigma- 
Aldrich, USA) was stored at a concentration of $10 \mathrm{mM}$ in DMSO at $-20^{\circ} \mathrm{C}$. All cells were maintained in a humidified incubator at $37^{\circ} \mathrm{C}$ with $5 \%$ carbon dioxide.

\section{RNA interference and plasmid transfection}

siRNAs (short interfering RNAs) and plasmids were transfected into cells with Lipofectamine 3000 (Invitrogen, USA) with or without P3000 (Invitrogen, USA) following the manufacturer's instructions. FGFR1 siRNA and an FGFR1 overexpression plasmid were constructed by our team previously. YAP siRNA, a YAP overexpression plasmid and two PD-L1 overexpression plasmids were purchased from Shanghai Huzbio Biotechnology (China). A PD-L1 promoter luciferase plasmid expressing firefly and Renilla was overexpressed. The sequences were as follows: siYAP-1: sense 5'- GGUGAUACUAUCAACCAAAdTdT -3', antisense 5'- UUUGGUUGAUAGUAUCACCdTdT -3'; siYAP-2: sense 5'- AGAUACUUCUUAAAUCACAdTdT -3', antisense 5'- UGUGAUUUAAGAAGUAUCUdTdT - 3 '; siRNA-NC: sense 5'- GCCACCAAGCUAGAUAAAGAAdTdT -3', antisense 5'- UUCUUUAUCUAGCUUGGUGGCdTdT -3'; YAP-OE:

forward: 5'- CGCAAATGGGCGGTAGGCGTG -3', reverse: 5'-AGTCCCGTCCTAAAATGTC -3';

PD-L1-OE:

forward: 5'- CGCAAATGGGCGGTAGGCGTG -3', reverse: 5'-AGTCCCGTCCTAAAATGTC -3';

PD-L1-Luc: forward: 5'- CGCCAGTCAAGTAACAAC -3', reverse: 5'-TTCCACACCCTAACTGACAC -3'.

\section{Lentivirus transduction and generation of stable cell lines}

Human FGFR1 lentivirus and a negative control carrying GFP and a puromycin tag were provided by Shanghai Huzbio Biotechnology (China). Recombinant lentiviral vectors containing mouse FGFR1 shRNA and irrelevant sequences were purchased from Shanghai DANGEN Biotechnology (China). In addition to the lentivirus expression vectors, a vector expressing a green fluorescent protein (GFP) and a hygromycin B reporter gene driven by the CMV promoter was used. Human and mouse transduction and stable cell line selection were performed according to the manufacturers' instructions. The sequences were as follows:

Human shFGFR1-1: CCGGGAGATGGAGGTGCTTCACTTACTCGAGTAAGTGAAGCACCTCCATCT CTTTTTG, Human shFGFR1-2: CCGGTCTTGAAGACTGCTGGAGTTACTCGAGTAACTCCAGCAGTCTTCAAG 
ATTTTTG.

Mouse shFGFR1-1:

GATGTTGAAGTCCGACGCACTCGAGTGCGTCGGACTTCAACATCTTTTT, Mouse shFGFR1-2:

CCGGCCTGGAGCATCATAATGGATTCTCGAGAATCCATTATGATGCTCCAGG TTTTT,

Mouse shFGFR1-3:

CCAGATCCTGAAGACTGCTGGAGTTCTCGAGAACTCCAGCAGTCTTCAGG ATCTGGTTTTT.

\section{Western blot analysis, RNA extraction, cDNA synthesis, and qPCR}

Cells were lysed for protein or RNA extraction, and the samples were subjected to western blot analysis or used for cDNA synthesis and qPCR as previously described (5). The primers used for qPCR were as follows:

\section{FGFR1:}

forward 5'- TAATGGACTCTGTGGTGCCCTC -3', reverse 5'- ATGTGTGGTTGATGCTGCCG -3';

YAP:

forward 5'- CATGTTCGAGCTCATGCCTCTCCAGCTTCTCTG -3', reverse 5'- CAGAGAAGCTGGAGAGGCATGAGCTCGAACATG -3';

TAZ:

forward 5'- GTATCCCAGCCAAATCTCGTGATG -3', reverse 5'- CAGCGCATTGGGCATACTCATG -3';

PD-L1:

forward 5'- GGTGCCGACTACAAGCGAAT-3', reverse 5'- AGCCCTCAGCCTGACATGTC -3'; GAPDH:

forward 5'- ACAACTTTGGTATCGTGGAAGG', reverse 5'- GCCATCACGCCACAGTTTC -3'.

\section{Immunoblotting}

Cell lysates were eluted with SDS-loading buffer (50 mM Tris-HCl, $\mathrm{pH}$ 6.8, 10\% glycerol, $1 \%$ SDS, $1 \%$ beta-mercaptoethanol). The eluates were separated by SDSPAGE and transferred to PVDF membranes (EMD Millipore, Germany). the antibodies included FGF Receptor 1 (D8E4, CST, USA), phospho-FGF Receptor 1 (Tyr653/654) (D4X3D, CST, USA), LATS1 (C66B5, CST, USA), phospho-LATS1/2 (Ser909/Ser872, Affinity, China), phospho-LATS1 (Thr1079, Affinity, China), YAP (D8H1X, CST, USA), phospho-YAP (Ser127) (D9W2I, CST, USA), YAP/TAZ (D24E4, CST, USA), PD-L1 (E1L3N, CST, USA), and GAPDH (14C10, CST, USA). Western blotting images were captured with a ChemiScope5600 instrument (Clinx, Shanghai, China) using ECL substrate.

\section{Dual luciferase reporter assay}

H520 cells were seeded in a 24-well plate until the cells reached approximately 50-60\% 
confluence. For the luciferase assay, the cells were transiently transfected with the indicated plasmids, and Renilla and firefly luciferase were used as internal controls. The cells were lysed with passive lysis buffer (Promega, USA) 48 hours after transfection and the indicated treatment. Luciferase assays were performed using a dual luciferase assay kit (Promega, USA), and luciferase activity was normalized to that of the internal control Renilla.

\section{Chromatin immunoprecipitation (ChIP)}

Briefly, chromatin samples were incubated with specific antibodies in ChIP lysis buffer (20 mM Tris- $\mathrm{HCl}$ (pH 8.1), $150 \mathrm{mM} \mathrm{NaCl}, 2$ mM EDTA, 1\% Triton X-100 and 0.05\% SDS) overnight at $4{ }^{\circ} \mathrm{C}$. The experiment was performed as previously reported (29). Protein-DNA complexes were immobilized on prewashed protein A/G beads ( $20 \mu \mathrm{L}$ per reaction). The bound fractions were washed 3 times with lysis buffer, twice with lowsalt wash buffer (10 mM Tris-HCl, $250 \mathrm{mM} \mathrm{LiCl,} 1 \mathrm{mM}$ EDTA, $0.5 \% \mathrm{NP}-40,0.5 \% \mathrm{Na}-$ deoxycholate), and once with $10 \mathrm{mM}$ Tris- $\mathrm{HCl}(\mathrm{pH} 8.0)$. Elution and reverse crosslinking were carried out in elution buffer (50 mM Tris- $\mathrm{HCl}(\mathrm{pH} \mathrm{8.0)}$, and 1\% SDS) at $65^{\circ} \mathrm{C}$ for 5 hours. After treatment with RNase $\mathrm{A}(1 \mathrm{unit} / \mu \mathrm{L})$ at $37^{\circ} \mathrm{C}$ for 1 hour and digestion with proteinase $\mathrm{K}(1 \mathrm{unit} / \mu \mathrm{L})$ at $55^{\circ} \mathrm{C}$, the DNA samples were purified using a PCR extraction kit (QIAGEN \#28006, USA). The precipitated DNA samples were either analyzed by qPCR or prepared for DNA deep sequencing according to the manufacturer's guidelines (Vazyme \#ND607, USA).

\section{Coculture of cancer cells and $T$ cells}

Jurkat T cells were activated with $100 \mathrm{UI} / \mathrm{ml}$ IL-2 3 days before the experiment. Cancer cells were inoculated in 24-well plates or 96-well plates 2 days in advance. Complete culture medium containing $500 \mathrm{IU} / \mathrm{ml} \mathrm{IFN-} \gamma$ was added to H520 and HCC 95 cancer cells for 24 hours to induce PD-L1 expression. Suspended/adherent cells were added to the coculture system at a ratio of 2:1 to $8: 1$ and cultivated for 48$72 \mathrm{~h}$. A total of $1.0 \times 10^{4}$ cells were seeded in $500 \mu \mathrm{L}$ medium in each well $(\mathrm{N}=3)$ of a 24-well plate. A total of $2.5 \times 10^{3}$ cells were seeded in $100 \mu \mathrm{L}$ medium in each well $(\mathrm{N}=5)$ of a 96-well plate. Then, Jurkat $\mathrm{T}$ cells were isolated from the culture supernatant for flow cytometry analysis, and adherent cancer cells were washed with PBS twice for crystal violet staining or the CCK-8 assay.

\section{Viability of cancer cells}

Surviving H520 or HCC95 cells in 24-well plates were fixed and stained with crystal violet solution, and pictures of the dried plates were taken. The viability of residual tumor cells was also tested with a Cell Counting Kit-8 (Dojindo Co., Japan) according to the manufacturer's protocol. The viability of the vehicle group was set as $100 \%$. The cell survival rate $(\%)$ was calculated as (OD value of treatment group/OD value of vehicle group) $\times 100 \%$. The data are presented as the mean $\pm \mathrm{SD}$ and were graphed with Prism software (7.0, GraphPad Software).

\section{Flow cytometry (FCM)}


Cells from the coculture system or those obtained by tumor digestion were resuspended in PBS/EDTA/BSA containing Fc Block (BD Biosciences, USA), centrifuged, incubated for 30 min with surface marker antibodies, washed with PBS/EDTA/BSA and then resuspended in $300 \mu \mathrm{L}$ PBS/EDTA/BSA. For cytokine amplification and detection, cells were cultured with Cell Activation Cocktail (with Brefeldin A) for 6 hours, processed with the Foxp3/Transcription Factor Staining Buffer Set (eBioscience, USA) according to the manufacturer's instructions and then intracellularly stained. Human and mouse antibodies were purchased from Biolegend (USA) and BD Pharmingen (USA). All the stained cells were analyzed using a BD Fortessa flow cytometer. The data were analyzed using FlowJo V10.0.7 software.

\section{In vivo subcutaneous lung cancer model}

The mouse NSCLC cell line LLC was obtained from our laboratory. Hygromycin B (1 $\mu \mathrm{g} / \mathrm{ml}$ ) was added to shFGFR1 and the control shRNA. Healthy male C57BL/6 mice aged 6-8 w were inoculated with FGFR1 knockdown or wild-type LLC cells. The cells $\left(50^{*} 10^{\wedge} 4\right)$ were digested, washed, and resuspended in $100 \mu \mathrm{L}$ PBS before being injected into the left armpit of each mouse. Beginning on day 6 , the size of each tumor was measured with calipers every three days. The subcutaneous tumor volume was calculated as $(\mathrm{L} 2 \times \mathrm{W}) / 2$, where $\mathrm{V}$ is the tumor volume, $\mathrm{L}$ is the longest diameter, and $\mathrm{W}$ is the shortest diameter. InVivoMab anti-mouse PD-1 (Bio X Cell, USA) or rat IgG2 $\alpha$ isotype (Bio X Cell, USA) was injected intraperitoneally at a dose of $100 \mu \mathrm{g}$ per mouse on day $6,8,10$ and 12 . The mice were sacrificed on day 15 . The tumors were removed and then imaged and analyzed. The tissues were digested with collagenase A $(0.33 \mathrm{U} / \mathrm{ml})$, dispase $(0.85 \mathrm{U} / \mathrm{ml})$ and DNase I $(144 \mathrm{U} / \mathrm{ml})$ with rapid shaking at $37^{\circ} \mathrm{C}$. Large pieces of debris were removed by successive filtration through $70-\mu \mathrm{m}$ and $40-\mu \mathrm{m}$ strainers.

\section{Immunofluorescence (IF) and immunohistochemistry (IHC)}

Tumors were fixed and embedded in paraffin after being removed. Sections were cut at a thickness of $10 \mu \mathrm{m}$ and subjected to Opal multiple fluorescence staining as previously described (30). The sections were incubated with DAPI (4',6-diamidino-2phenylindole, Dilactate, Biolegend), an FGF Receptor 1 antibody (D8E4, CST, USA), a YAP antibody (D8H1X, CST, USA) and a PD-L1 antibody (E1L3N, CST, USA). Other sections were deparaffinized, subjected to antigen retrieval and immunostained with a ki-67 antibody (Servicebio, GB111141, 1:500, China) and CD8 antibody (Abcam, ab237723, England) as previously described (31). The stained cells were observed and imaged under an immunofluorescence microscope (Leica DFC420C, Germany).

\section{Bioinformatics analysis of public datasets}

Relative FGF2 and PD-L1 copy number and mRNA level data from provisional LSQCC cohorts in public datasets from The Cancer Genome Atlas (TCGA) and Kaplan-Meier Plotter database were downloaded from cBioPortal. The data obtained from our institution was from 37 LSQCC patients and were reported previously (32). 
Linear regression and Spearman correlation analysis of the mRNA levels of FGF2 and PD-L1 was conducted as previously reported (5). The log-rank test was used for statistical analysis. Single-cell RNA-seq gene expression data for tumor tissues from NSCLC patients were downloaded from Gene Expression Omnibus (GEO). The accession number of the dataset was GSE140819 (33). Seurat 4.0.1 was used to standardize, integrate, cluster, visualize, and annotate the original data using an $\mathrm{R}$ tool kit for single-cell genomics $(33,34)$.

\section{Statistical analysis}

Statistical analyses were performed in Prism software (version 7.0a, GraphPad Software Inc.) and R software (version 4.0.3). Two-tailed, unpaired Student's t-test and Welch's correction were used. For multiple comparisons, one-way ANOVA followed by Bonferroni's multiple comparisons test was performed. P-values $<0.05$ were considered significant $(* \mathrm{p}<0.05, * * \mathrm{p}<0.01, * * * \mathrm{p}<0.001, * * * * \mathrm{p}<0.0001)$. Except where otherwise indicated, the experiments were repeated three times. Quantitative data are presented as the mean \pm SEM. All images shown are representative.

\section{Results \\ FGFR1 regulated PD-L1 expression through YAP in LSQCC}

In $\mathrm{H} 520$ and HCC95 cells, protein and mRNA expression was measured after treatment with small interfering RNAs (siFGFR1-1, siFGFR1-2 or siCTRL; constructed and verified as previously reported (5)), an FGFR1/2/3 inhibitor (AZD4547) and an FGFR1 activator (bFGF2). siFGFR1 and AZD4547 inhibited FGFR1, pFGFR1 and PD-L1 expression (Figure 1A and B). There was no difference in LATS1 expression, but the phosphorylation of LATS1 at two amino acid residues (S909 and T1079) was decreased after FGFR1 inhibition (Figure 1A and B). As demonstrated previously, a decrease in phospho-LATS1 expression facilitated the phosphorylation of YAP at the S127 residue. When trapped in the cytoplasm, YAP was inactivated through phosphorylation (Figure 1A and B). The total mRNA levels and protein levels of FGFR1, YAP, TAZ and PD-L1 were similar (Figure 1C and D). During FGFR1 inhibition, less YAP was able to enter the nucleus, thus reducing the transcription levels of downstream molecules. On the other hand, PD-L1 was activated in a time-dependent manner by FGF2, consistent with YAP activation (Figure 1E).

Next, we investigated the underlying mechanism. A small interfering RNA targeting YAP was constructed, and its efficacy was verified by western blotting. siYAP inhibited the expression of YAP and PD-L1 (Figure 2A). A plasmid containing PD-L1 promoter region with a fluorescent label was first transfected into cells. Then, a YAPoverexpressing (YAP-OE) plasmid was added to the cells for YAP and PD-L1 amplification. The fluorescence intensity of the cancer cells was determined 48 hours later, and it was found to be increased in the YAP-OE group $(p<0.001)$ (Figure 2B). This indicated that YAP acted on the promoter region of PD-L1. To confirm this, H520 cells were cultured with IFN- $\gamma$ to stimulate the expression of PD-L1 and then lysed, and DNA was extracted. Subsequently, chromatin immunoprecipitation was performed to 
analyze the cDNA library. The chromatin region precipitated by the YAP antibody was located between 5,440,000 and 5,460,000 bases in the PD-L1 region of chromosome 9. Two known TEAD binding sites were differentially enriched in this region (Figure 2C).

To confirm the existence of the FGFR1/YAP/PD-L1 regulatory axis, siFGFR1-1 and YAP-OE plasmids were used. Overexpression of YAP reversed the expression of PD-L1 after FGFR1 inhibition (Figure 2D). Conversely, an FGFR1 overexpression plasmid (FGFR1-OE) and siYAP-2 were used, and it was found that PD-L1 expression was also reversed by siYAP-2 after FGFR1-OE (Figure 2E).

In summary, FGFR1 was shown to positively regulate PD-L1 expression through YAP in H520 and HCC95 cells, and YAP promoted PD-L1 transcription by binding to the promoter region directly. Considering the results shown in Figures 1 to 3 together, the FGFR1/YAP/PD-L1 axis promotes the immune escape of LSQCC in vitro.

\section{FGFR1 promoted tumor immune escape in the coculture system}

To construct stable FGFR1 knockdown strains, H520 and HCC95 cells were transfected with shFGFR1-1, shFGFR1-2 or shCTRL, and then the effects of the shRNAs were verified by western blotting and qPCR (Figure 3A and B). shFGFR1 decreased protein and mRNA levels in H520 and HCC95 cells, and sequence 1 was chosen for subsequent study.

To mimic the interaction between cancer cells and immune cells, a coculture system was established. Suspended Jurkat T cells were added to adherent shFGFR1 or shCTRL cancer cells treated with a human PD-1 antibody (nivolumab) or control antibody (isotype $\operatorname{IgG}$ ). After coculture for 72 hours, the suspended cells were removed, and cell proliferation was assessed by crystal violet staining and the Cell Counting Kit8 (CCK-8) assay. Cell viability was reduced when FGFR1 was knocked down and was mostly decreased when FGFR1 knockdown was combined with nivolumab treatment (Figure 3C and D). FGFR1 knockdown inhibited cell proliferation in the absence of Jurkat T cells, as was previously reported (5). The toxicity of nivolumab to H520 and HCC95 cells outside of a coculture system was limited, as previously reported (35).

Jurkat $\mathrm{T}$ cells were removed and stained for immune evasion markers and immunestimulating cytokines after coculture. In the experimental group (coculture with FGFR1 knockdown cancer cells), the expression of PD-1 and lymphocyte activation gene-3 (LAG-3) was decreased significantly $(\mathrm{p}<0.0001)$, while the expression of interleukin2 (IL-2) was increased $(\mathrm{p}<0.05)$. There was no significant difference in the expression of interferon-gamma (IFN- $\gamma)(p=0.7267)$ or tumor necrosis factor-alpha $(\mathrm{TNF}-\alpha)(\mathrm{p}=$ 0.2386) (Figure 3E). These results indicated that FGFR1 inhibition partially reversed tumor immune escape in vitro. In other words, if FGFR1 is activated, FGFR1 might promote immune evasion in addition to causing the known phenotypes observed previously. These results were further confirmed in a mouse model.

\section{FGFR1 knockdown remodeled the tumor microenvironment and exerted antitumor effects in combination with PD-1 antibodies}

The interaction between cancer cells and surrounding immune cells is conducive to the growth and development of cancer $(36,37)(38)$. To explore the effect of FGFR1 
on the tumor microenvironment (TME), stable FGFR1 knockdown (shFGFR1) or FGFR1 wild-type (shCTRL) Lewis lung carcinoma (LLC) strains were constructed by shRNA transfection and analyzed by western blotting. Lentivirus sequence 1 showed the strongest inhibitory effect on FGFR1 and PD-L1 expression and thus was amplified later for tumor inoculation (Figure 4A). C57BL/6 mice were injected with shFGFR1or shCTRL-transfected LLC cells and injected with a mouse PD-1 monoclonal antibody (PD-1 mAb) or a control antibody (IgG), as shown in the flowchart (Figure 4B).

Photographs and growth curves of the tumors showed that the mouse PD-1 antibody alone only slightly inhibited tumor growth $(p=0.0149)$, while FGFR1 inhibition reduced the tumor volume more $(p<0.0001)$. In the two groups inoculated with the shFGFR1-transfected cell strains, the PD-1 antibody showed a synergistic inhibitory effect in combination with FGFR1 knockdown, as indicated by Bonferroni's multiple comparisons test $(p=0.0156)$. Therefore, FGFR1 knockdown combined with PD-1 antibody treatment mostly inhibited tumor growth $(\mathrm{p}<0.0001)$ (Figure 4C and D).

Tumor tissues were embedded in paraffin and sectioned. Immunofluorescence staining showed that the expression levels of FGFR1, YAP and PD-L1 in the TME decreased after FGFR1 knockdown (Figure 4E). The trend in expression was consistent with what was observed in vitro (Figure 1A-D). Then, Ki-67 and CD8 were detected by immunohistochemistry. After FGFR1 knockdown, cancer cell proliferation decreased, as indicated by ki-67 staining (Figure 4F), while the infiltration of CD8+ T cells increased (Figure 4G).

After the mice were sacrificed, another part of the tumor tissue was processed for flow cytometry. Single-cell suspensions were stained with antibodies against different cell surface markers and cytokines as previously described (31). The analysis showed that FGFR1 knockdown increased the number of CD8+ T cells in the TME $(p=0.005)$ (Figure 5A). Further analysis of the CD8+ subset showed that the expression of the immune escape marker PD-1 was decreased $(p=0.0057)$ (Figure 5B) but that there was no significant difference in LAG-3 expression $(p=0.5992)$ (Figure 5C); however, the expression of the immune activating cytokines IL-2 $(p=0.0041)$ (Figure 5D), IFN- $\gamma(p$ $=0.0034)($ Figure 5E) and TNF- $\alpha(p=0.0042)($ Figure 5F) was increased. These results suggested that FGFR1 knockdown increased the number of, partially reduced the immune escape of, and reactivated the tumor killing ability of CD8+ T cells in the TME.

To comprehensively investigate the effect of FGFR1 on the immune profile of the TME, we detected immune cells other than CD8+ T cells. It is worth noting that the number of M1-like tumor-associated macrophages (M1-TAMs) (CD206+/CD45.2+MHCII+F480+) was significantly decreased $(\mathrm{p}<0.0001)$ (Figure 5I) in the FGFR1 knockdown group compared with the control group. Classically activated macrophages promote the secretion of a variety of inflammatory cytokines and mediate the killing of microorganisms and tumor cells; thus, they have the opposite effects of M2-TAMs (39). After FGFR1 knockdown, the number of CD8+ T cells was increased and the number of M1-TAMs was decreased simultaneously, and the changes in the number of these cells played an opposite role in the antitumor effect. This might be one of the reasons why inhibition of FGFR1 alone was not found to be efficacious 
in current clinical trials, as the human body is much more complicated than the mouse. Although FGFR1 inhibition alone greatly reduced tumor growth in a mouse model, the complexity of human tumors needs to be understood more deeply.

There was no significant difference in the number of total macrophages $(\mathrm{F} 480+/ \mathrm{CD} 45.2+\mathrm{MHCII}+) \quad(\mathrm{p}=0.8291) \quad($ Figure $5 \mathrm{G}), \quad$ M2-TAMs $(\mathrm{CD} 80+/ \mathrm{CD} 45.2+\mathrm{MHCII}+\mathrm{F} 480+)(\mathrm{p}=0.8589)($ Figure $5 \mathrm{H})$ or $\mathrm{CD} 4+\mathrm{T}$ cells $(\mathrm{p}=$ 0.0973 ) (Figure $5 \mathrm{~J}$ ) between the two groups. In addition, there was only a slight decrease in the number of monocytes, including CD11b+/CD45.2+MHCII+ cells $(\mathrm{p}=$ 0.0047) (Figure 5K) and CD11c+/CD45.2+MHCII+ cells ( $\mathrm{p}=0.0287$ ) (Figure 5L), in the shFGFR1 group.

In this part of the mouse experiment, we found that FGFR1 inhibition increased the number of CD8+ T cells in the TME, inhibited immune escape, and stimulated the production of immunoreactive cytokines. Moreover, the decrease in the number of M1TAMs suggested that the effect of FGFR1 on the TME might be contradictory and complicated. Tumor growth was more robustly inhibited by FGFR1 knockdown in combination with PD-1 antibodies than by shFGFR1 alone or PD-1 mAbs alone. This combination is a promising therapeutic strategy for LSQCC patients harboring sensitive FGFR1 variations, although more research on the predictive biomarker is needed.

\section{FGFR1 expression was correlated with PD-L1 and FGFR1 was coexpressed with PD-L1 in patients with LSQCC}

To evaluate the correlation between FGFR1 and PD-L1 expression in LSQCC patients, we first analyzed the copy number and expression profiles of FGFR1 and PDL1 in our LSQCC cohort (32) and the provisional LSQCC cohort from TCGA. The results showed no statistical correlation between FGFR1 and PD-L1 expression (Figure $6 \mathrm{~A}$ and $\mathrm{C}$ ), which was similar to the results of a previous study (40). To our surprise, FGF2 expression and PD-L1 expression were positively correlated, and coamplification of PD-L1 at the mRNA level was common in LSQCC patients with FGF2 amplification (Figure 6B and D). Considering that FGF2 can activate FGFR1, these results suggest an inequality between FGFR1 amplification and FGFR1 downstream pathway activation, which might explain why FGFR1 amplification is not a sensitive predictive biomarker for FGFR1 inhibitors $(2,8)$.

We further investigated the distribution of these molecules in the TME due to intratumoral heterogeneity. Selected single-cell sequencing data from the TCGA database were analyzed and visualized with the Seurat package in $\mathrm{R}$ version 4.0.2. After data standardization and reduction, the 1500 genes with the largest variation among nearly 20,000 genes were group and annotated (41). Cells were groups according to molecular profiles by t-SNE reduction (42) (Supplementary Figure 1).

As shown in the bubble graph, FGF2, FGFR1, YAP and PD-L1 coexpressed mainly in endothelial cells and fibroblasts in the TME (Supplementary Figure 2A). This suggested that FGF2/FGFR1/YAP/PD-L1 might act as a regulatory axis, mainly in cancer cells in heterogeneous tumor tissues, according to the pathological characteristics of LSQCC. Markers were visualized by t-SNE in scattering mode (Supplementary Figure 2B). The FGFR1/YAP/PD-L1 regulatory axis existed in certain 
subsets and all cancer cells within the TME, indicating the heterogeneity of the different cell groups in just one tumor.

\section{Discussion}

FGFR1 variations are among the most common mutations in LSQCC patients $(43,44)$. However, ongoing clinical trials show that the response rate to FGFR1-targeted therapy is limited (Dai et al., 2015; Javle et al., 2018; Lim et al., 2016). There are many possible reasons for this. One possible explanation is that there are unknown phenotypes of FGFR1 variations, which contribute to carcinogenesis and allow a limited response to currently used drugs. In this study, we identified that the FGFR1/YAP/PD-L1 signaling pathway is involved in the tumorigenesis of LSQCC through immune evasion (Figure 7). In C57BL/6 mice, the paradoxical regulatory effects of FGFR1 on CD8+ T cells and M1-TAMs in the TME might hinder the antitumor effect of FGFR1-targeted therapies in the sophisticated immune environment. To address this problem, we tried a combination strategy and found that FGFR1 knockdown combined with anti-PD-1 therapy mostly inhibited tumor growth.

FGFR1 variations, such as FGFR1 amplification, have been used as predictive biomarkers in previous clinical trials $(47,48)$. Unfortunately, these biomarkers are not as sensitive as epidermal growth factor receptor (EGFR) mutations for lung adenocarcinoma (49). Interestingly, the expression of FGF2 mRNA, but not FGFR1 mRNA, correlated with the expression of PD-L1 mRNA (Figure 6). The heterogeneity between FGFR1 variations and FGFR1 pathway activation might indicate that FGFR1 amplification, fusion or point mutations are not effective predictive biomarkers because they do not indicate activation of downstream pathways of FGFR1 at the functional level (50). Higher FGF2 expression is more likely to indicate activation of the FGFR1 pathway, but this needs to be confirmed in clinical trials (1).

Several studies have shown the effect of pan-FGFR inhibitors on the TME in vivo. In a genetically engineered mouse model of primary KRAS-G12C mutant lung adenocarcinoma, the diversity of interacting T cell receptor (TCR) clones was shown to decrease after treatment with erdafitinib, while the number of TCR clones was shown to increase (51). In the Renca mouse renal carcinoma model, ODM-203 decreases PD1 expression in CD8+ T cells and the number of NK cells but increases the ratio of total $\mathrm{T}$ cells to Tregs, indicating that most of the $\mathrm{T}$ cells that are increased in number are effector T cells (52). In a mouse model of 4T1 breast cancer, PD173074 reduces the number of myeloid-derived suppressor cells (MDSCs) in the blood, spleen and tumor and increases the infiltration of CD4+ and CD8+ T cells into the spleen and tumor (20). In a mouse model of MC38 colorectal adenocarcinoma, 3D185 increases the number of activated CD $8+T$ cells (IFN- $\gamma+$ CD8 + T cells) while decreasing the number of TAMs and Tregs (17). Although the abovementioned studies investigated the immuneremodeling effects of FGFR inhibitors, none of them showed the exact influences of FGFR1 on the TME or the regulatory mechanism.

There are limitations to our work. For example, we revealed the ternary regulatory effect of FGFR1/YAP/PD-L1 and the specific site in the promoter region of PD-L1 to which YAP bound. However, it was not clear whether there are other molecules 
involved in the interaction between FGFR1 and PD-L1. Due to limitations related to time and cost, we did not construct a mouse model of primary LSQCC or a transplantation tumor model of LSQCC patient tissues. If possible, immune cells modulation should be combined with FGFR1 regulation in vivo to identify additional mechanisms.

\section{Conclusions}

In summary, we verified the positive regulatory effect of FGFR1 on PD-L1 expression in vitro and in vivo. Furthermore, YAP was confirmed to bind to the promoter region of PD-L1 to initiate the transcription of the latter. In the coculture system and subcutaneous tumor model, we found that FGFR1 knockout increased the antitumor activity of CD8 + T cells and decreased the expression level of immune escape-related molecules. FGFR1 knockdown in combination with PD-1 antibody treatment significantly suppressed tumor growth in vitro and in vivo. These results provide new insights into the function of FGFR1 related to tumor immune escape and preclinical evidence for potential targeted therapies and immunotherapy for LSQCC patients.

\section{Declarations}

\section{Ethics approval and consent to participate}

All animal experiments were carried out in full accordance with protocols approved by the Institutional Ethics Committee of Shanghai Jiao Tong University.

\section{Consent for publication}

Consent to publish has been obtained from all authors.

\section{Availability of supporting data}

The datasets used and/or analyzed during the current study are available from the corresponding author on reasonable request.

\section{Competing interests}

The authors declare no potential conflicts of interest.

\section{Funding}

This work was funded by the National Key R\&D Program of China (2016YFC1303300 to S.L), the National Natural Science Foundation of China Grants (81672272 to S.L), the Science and Technology Innovation program of Shanghai (19411950500 to S.L), Shanghai Municipal Science and Technology Commission Research Project (17431906103 to S.L.), Program for Outstanding Medical Academic Leader (to S.L), Shanghai Sailing Program (19YF1407200 to K. W), Shanghai Sailing Program (19YF1407300 to W.J).

\section{Authors' Contributions}

Conception and design: Shun Lu, Weiliang Xia, Min Lu and Kaixuan Wang. 
Acquisition of data: Min Lu and Kaixuan Wang.

2 Analysis and interpretation of data: Min Lu and Kaixuan Wang.

3 Writing, review, and/or revision of the manuscript: Min Lu, Kaixuan Wang, and

4 Wenxiang Ji.

5 Administrative, technical, or material support: Yongfeng Yu, Ziming Li, Lan Shen,

$6 \quad$ Weiliang Xia and Shun Lu.

7 Study supervision: Weiliang Xia and Shun Lu.

8 All authors read and approved the final manuscript.

\section{Acknowledgements}

We sincerely thank Haizhen Jin for the flow cytometry testing and guidance.

\section{Authors' information:}

Min $\mathrm{Lu}$ and Kaixuan Wang have contributed equally to this work.

\section{Affiliations}

Shanghai Lung Cancer Center, Shanghai Chest Hospital, Shanghai Jiao Tong

University, West Huaihai Road 241, Shanghai, 20030, China.

Min Lu, Kaixuan Wang, Wenxiang Ji, Yongfeng Yu, Ziming Li, Shun Lu

School of Biomedical Engineering and Med-X Research Institute, Shanghai Jiao Tong University, Huashan Road 1954, Shanghai, 200030, China.

Min Lu, Weiliang Xia

\section{Corresponding authors}

Correspondence to Weiliang Xia and Shun Lu.

\section{Conflict of interest}

The authors declare no conflict of interest.
Abbreviations
FGFR1 fibroblast growth factor receptor 1
LSQCC lung squamous cell carcinoma
PD-1 programmed death-1
PD-L1 programmed death ligand-1
YAP Yes-associated protein
FGF2 fibroblast growth factor 2
TKIs tyrosine kinase inhibitors
ICIs immune checkpoint inhibitors
DCs dendritic cells
TME tumor microenvironment
TEAD transcriptional enhanced associate domain
siRNAs short interfering RNAs
GFP green fluorescent protein 
1 ChIP chromatin immunoprecipitation

2 CCK-8 cell Counting Kit-8

3 FCM flow cytometry

4 IF immunofluorescence

5 IHC immunohistochemistry

6 TCGA The Cancer Genome Atlas

7 GEO Gene Expression Omnibus

8 LAG-3 lymphocyte activation gene-3

9 IL-2 interleukin-2

10 IFN- $\gamma$ interferon-gamma

11 TNF- $\alpha$ tumor necrosis factor-alpha

12 TME tumor microenvironment

13 M1-TAMs M1 like tumor associated macrophages

14 M2-TAMs M2 like tumor associated macrophages

15 EGFR epidermal growth factor receptor

16 TCR T cell receptor

17 MDSC myeloid-derived suppressor cells

\section{References}

1. Babina IS, Turner NC. Advances and challenges in targeting FGFR signalling in cancer. Nat Rev Cancer. 2017 May;17(5):318-32.

2. Weiss J, Sos ML, Seidel D, Peifer M, Zander T, Heuckmann JM, et al. Frequent and Focal FGFR1 Amplification Associates With Therapeutically Tractable FGFR1 Dependency in Squamous-cell Lung Cancer. Sci Transl Med. 2010 Dec 15;2(62):62ra93.

3. Heist RS, Mino-Kenudson M, Sequist LV, Tammireddy S, Morrissey L, Christiani DC, et al. FGFR1 amplification in squamous cell carcinoma of the lung. J Thorac Oncol. 2012 Dec;7(12):1775-80.

4. Desai A, Adjei AA. FGFR Signaling as a Target for Lung Cancer Therapy. J Thorac Oncol. 2016 Jan 1;11(1):9-20.

5. Wang K, Ji W, Yu Y, Li Z, Niu X, Xia W, et al. FGFR1-ERK1/2-SOX2 axis promotes cell proliferation, epithelial-mesenchymal transition, and metastasis in FGFR1-amplified lung cancer. Oncogene. 2018 Sep;37(39):5340-54.

6. Lu T, Li Z, Yang Y, Ji W, Yu Y, Niu X, et al. The Hippo/YAP1 pathway interacts with FGFR1 signaling to maintain stemness in lung cancer. Cancer Lett. 2018 Jun;423:36-46.

7. Quintanal-Villalonga A, Molina-Pinelo S, Cirauqui C, Ojeda-Márquez L, Marrugal Á, Suarez $\mathrm{R}$, et al. FGFR1 cooperates with EGFR in lung cancer oncogenesis, and their combined inhibition shows improved efficacy. J Thorac Oncol. 2019 Jan 9;14(4):641-55.

8. Pearson A, Smyth E, Babina IS, Herrera-Abreu MT, Tarazona N, Peckitt C, et al. High-Level Clonal FGFR Amplification and Response to FGFR Inhibition in a Translational Clinical Trial. Cancer Discov. 2016 Aug;6(8):838-51.

9. Paik PK, Shen R, Berger MF, Ferry D, Soria J-C, Mathewson A, et al. A Phase 1b Open Label Multicentre Study of AZD4547 in Patients with Advanced Squamous Cell Lung Cancers. Clin Cancer Res Off J Am Assoc Cancer Res. 2017 Sep 15;23(18):5366-73.

10. Aggarwal C, Redman MW, Lara PN, Borghaei H, Hoffman P, Bradley JD, et al. SWOG 
S1400D (NCT02965378), a Phase II Study of the Fibroblast Growth Factor Receptor Inhibitor AZD4547 in Previously Treated Patients With Fibroblast Growth Factor Pathway-Activated Stage IV Squamous Cell Lung Cancer (Lung-MAP Substudy). J Thorac Oncol. 2019 Oct;14(10):1847-52.

11. Lim SH, Sun J-M, Choi Y-L, Kim HR, Ahn S, Lee JY, et al. Efficacy and safety of dovitinib in pretreated patients with advanced squamous non-small cell lung cancer with FGFR1 amplification: A single-arm, phase 2 study. Cancer. 2016;122(19):3024-31.

12. Pardoll DM. The blockade of immune checkpoints in cancer immunotherapy. Nat Rev Cancer. 2012 Apr;12(4):252-64.

13. Song S, Yuan P, Wu H, Chen J, Fu J, Li P, et al. Dendritic cells with an increased PD-L1 by TGF- $\beta$ induce $T$ cell anergy for the cytotoxicity of hepatocellular carcinoma cells. Int Immunopharmacol. 2014 May 1;20(1):117-23.

14. Zhang X, Zeng Y, Qu Q, Zhu J, Liu Z, Ning W, et al. PD-L1 induced by IFN- $\gamma$ from tumorassociated macrophages via the JAK/STAT3 and PI3K/AKT signaling pathways promoted progression of lung cancer. Int J Clin Oncol. 2017 Dec 1;22(6):1026-33.

15. Jiang P, Gu S, Pan D, Fu J, Sahu A, Hu X, et al. Signatures of T cell dysfunction and exclusion predict cancer immunotherapy response. Nat Med. 2018 Oct;24(10):1550-8.

16. DeBerardinis RJ. Tumor Microenvironment, Metabolism, and Immunotherapy. Phimister EG, editor. N Engl J Med. 2020 Feb 27;382(9):869-71.

17. Peng X, Hou P, Chen Y, Dai Y, Ji Y, Shen Y, et al. Preclinical evaluation of 3D185, a novel potent inhibitor of FGFR1/2/3 and CSF-1R, in FGFR-dependent and macrophage-dominant cancer models. J Exp Clin Cancer Res. 2019 Dec;38(1):372.

18. Palakurthi S, Kuraguchi M, Zacharek SJ, Zudaire E, Huang W, Bonal DM, et al. The combined effect of FGFR inhibition and PD-1 blockade promotes tumor-intrinsic induction of antitumor immunity. Cancer Immunol Res. 2019 Jan 1;7(8):1457-71.

19. Li P, Huang T, Zou Q, Liu D, Wang Y, Tan X, et al. FGFR2 Promotes Expression of PD-L1 in Colorectal Cancer via the JAK/STAT3 Signaling Pathway. J Immunol. 2019 May 15;202(10):3065-75.

20. Ye T, Wei X, Yin T, Xia Y, Li D, Shao B, et al. Inhibition of FGFR signaling by PD173074 improves antitumor immunity and impairs breast cancer metastasis. Breast Cancer Res Treat. $2014 \mathrm{Feb} ; 143(3): 435-46$.

21. Dey A. Targeting the Hippo pathway in cancer, fibrosis, wound healing and regenerative medicine. Nat Rev Drug Discov. 19:480-94.

22. Zanconato F, Forcato M, Battilana G, Azzolin L, Quaranta E, Bodega B, et al. Genome-wide association between YAP/TAZ/TEAD and AP-1 at enhancers drives oncogenic growth. Nat Cell Biol. 2015 Sep;17(9):1218-27.

23. Meng Z, Moroishi T, Guan K-L. Mechanisms of Hippo pathway regulation. Genes Dev. 2016 Jan 1;30(1):1-17.

24. Feng J, Yang H, Zhang Y, Wei H, Zhu Z, Zhu B, et al. Tumor cell-derived lactate induces TAZ-dependent upregulation of PD-L1 through GPR81 in human lung cancer cells. Oncogene. 2017 Oct;36(42):5829-39.

25. Lee BS, Park DI, Lee DH, Lee JE, Yeo M, Park YH, et al. Hippo effector YAP directly regulates the expression of PD-L1 transcripts in EGFR-TKI-resistant lung adenocarcinoma. Biochem Biophys Res Commun. 2017 Sep 16;491(2):493-9. 
26. Miao J, Hsu P-C, Yang Y-L, Xu Z, Dai Y, Wang Y, et al. YAP regulates PD-L1 expression in human NSCLC cells. Oncotarget. 2017 Dec 9;8(70):114576-87.

27. Kim MH, Kim CG, Kim S-K, Shin SJ, Choe EA, Park S-H, et al. YAP-Induced PD-L1 Expression Drives Immune Evasion in BRAFi-Resistant Melanoma. Cancer Immunol Res. 2018 Mar 1;6(3):255-66.

28. Liang J, Wang L, Wang C, Shen J, Su B, Marisetty AL, et al. Verteporfin Inhibits PD-L1 through Autophagy and the STAT1-IRF1-TRIM28 Signaling Axis, Exerting Antitumor Efficacy. Cancer Immunol Res. 2020 Jul 1;8(7):952-65.

29. Grzybowski AT, Shah RN, Richter WF, Ruthenburg AJ. Native Internally Calibrated Chromatin Immunoprecipitation for Quantitative Studies of Histone Post-Translational Modifications. Nat Protoc. 2019 Dec;14(12):3275-302.

30. Schalper KA, Carvajal-Hausdorf D, McLaughlin J, Altan M, Velcheti V, Gaule P, et al. IF Differential Expression and Significance of PD-L1, IDO-1, and B7-H4 in Human Lung Cancer. Clin Cancer Res. 2017 Jan 15;23(2):370-8.

31. Vartuli RL, Zhou H, Zhang L, Powers RK, Klarquist J, Rudra P, et al. Eya3 promotes breast tumor-associated immune suppression via threonine phosphatase-mediated PD-L1 upregulation. J Clin Invest. 128(6):2535-50.

32. Tan Q, Li F, Wang G, Xia W, Li Z, Niu X, et al. Identification of FGF19 as a prognostic marker and potential driver gene of lung squamous cell carcinomas in Chinese smoking patients. Oncotarget. 2016 Apr 5;7(14):18394-402.

33. Slyper M, Porter CBM, Ashenberg O, Waldman J, Drokhlyansky E, Wakiro I, et al. sc-seq A single-cell and single-nucleus RNA-Seq toolbox for fresh and frozen human tumors. Nat Med. 2020 May;26(5):792-802.

34. Maynard A, McCoach CE, Rotow JK, Harris L, Haderk F, Kerr DL, et al. Therapy-Induced Evolution of Human Lung Cancer Revealed by Single-Cell RNA Sequencing. Cell. 2020 Sep 3;182(5):1232-1251.e22.

35. Wang C, Thudium KB, Han M, Wang X-T, Huang H, Feingersh D, et al. In vitro characterization of the anti-PD-1 antibody nivolumab, BMS-936558, and in vivo toxicology in non-human primates. Cancer Immunol Res. 2014 Sep;2(9):846-56.

36. Joyce JA, Pollard JW. Microenvironmental regulation of metastasis. Nat Rev Cancer. 2009 Apr;9(4):239-52.

37. Riaz N, Havel JJ, Makarov V, Desrichard A, Urba WJ, Sims JS, et al. Tumor and Microenvironment Evolution during Immunotherapy with Nivolumab. Cell. 2017 Nov 2;171(4):934-949.e16.

38. Church SE, Galon J. Tumor Microenvironment and Immunotherapy: The Whole Picture Is Better Than a Glimpse. Tumor Microenviron Immunother Whole Pict Better Glimpse. 2015 Oct 20;43(4):631-3.

39. Gordon SR, Maute RL, Dulken BW, Hutter G, George BM, McCracken MN, et al. PD-1 expression by tumour-associated macrophages inhibits phagocytosis and tumour immunity. Nature. 2017 May;545(7655):495-9.

40. Guo Q, Sun Y, Yu S, Bai H, Zhao J, Zhuo M, et al. Programmed cell death-ligand 1 (PD-L1) expression and fibroblast growth factor receptor 1 (FGFR1) amplification in stage III/IV lung squamous cell carcinoma (SQC). Thorac Cancer. 2017 Mar;8(2):73-9.

41. $\mathrm{Xu} \mathrm{C}, \mathrm{Su} \mathrm{Z}$. Identification of cell types from single-cell transcriptomes using a novel 
clustering method. Bioinformatics. 2015 Jun 15;31(12):1974-80.

42. Calles A, Riess JW, Brahmer JR. Checkpoint Blockade in Lung Cancer With Driver Mutation: Choose the Road Wisely. Am Soc Clin Oncol Educ Book. 2020 May;(40):372-84.

43. Helsten T, Elkin S, Arthur E, Tomson BN, Carter J, Kurzrock R. The FGFR Landscape in Cancer: Analysis of 4,853 Tumors by Next-Generation Sequencing. Clin Cancer Res. 2016 Jan 1;22(1):259-67.

44. Katoh M. Therapeutics Targeting FGF Signaling Network in Human Diseases. Trends Pharmacol Sci. 2016 Dec 1;37(12):1081-96.

45. Dai W, Luo B, Wu Z, Chen J, Feng G, Guan P. A multi-center phase II study of nintedanib as second-line therapy for patients with advanced non-small-cell lung cancer in China. Am J Cancer Res. 2015 Sep 15;5(10):3270-5.

46. Javle M, Lowery M, Shroff RT, Weiss KH, Springfeld C, Borad MJ, et al. Phase II Study of BGJ398 in Patients With FGFR-Altered Advanced Cholangiocarcinoma. J Clin Oncol. 2018 Jan 20;36(3):276-82.

47. Aggarwal C, Redman MW, Lara PN, Borghaei H, Hoffman P, Bradley JD, et al. Brief Report: SWOG S1400D (), a Phase II Study of the Fibroblast Growth Factor Receptor Inhibitor AZD4547 in Previously-treated Patients with Fibroblast Growth Factor Pathway-Activated Stage IV Squamous Cell Lung Cancer (Lung-MAP Sub-Study). J Thorac Oncol Off Publ Int Assoc Study Lung Cancer. 2019 Oct;14(10):1847-52.

48. Nogova L, Sequist LV, Perez Garcia JM, Andre F, Delord J-P, Hidalgo M, et al. Evaluation of BGJ398, a Fibroblast Growth Factor Receptor 1-3 Kinase Inhibitor, in Patients With Advanced Solid Tumors Harboring Genetic Alterations in Fibroblast Growth Factor Receptors: Results of a Global Phase I, Dose-Escalation and Dose-Expansion Study. J Clin Oncol. 2016 Nov 21;35(2):157-65.

49. Ramalingam SS, Vansteenkiste J, Planchard D, Cho BC, Gray JE, Ohe Y, et al. Overall Survival with Osimertinib in Untreated, EGFR-Mutated Advanced NSCLC. N Engl J Med. 2020 Jan 2;382(1):41-50.

50. Malchers F, Ercanoglu M, Schütte D, Castiglione R, Tischler V, Michels S, et al. Mechanisms of Primary Drug Resistance in FGFR1-Amplified Lung Cancer. Clin Cancer Res. 2017 Sep 15;23(18):5527-36.

51. Loriot Y, Necchi A, Park SH, Garcia-Donas J, Huddart R, Burgess E, et al. Erdafitinib in Locally Advanced or Metastatic Urothelial Carcinoma. N Engl J Med. 2019;381(4):338-48.

52. Holmström TH, Moilanen A-M, Ikonen T, Björkman ML, Linnanen T, Wohlfahrt G, et al. ODM-203, a Selective Inhibitor of FGFR and VEGFR, Shows Strong Antitumor Activity, and Induces Antitumor Immunity. Mol Cancer Ther. 2019 Jan;18(1):28-38. 
Fig. 1

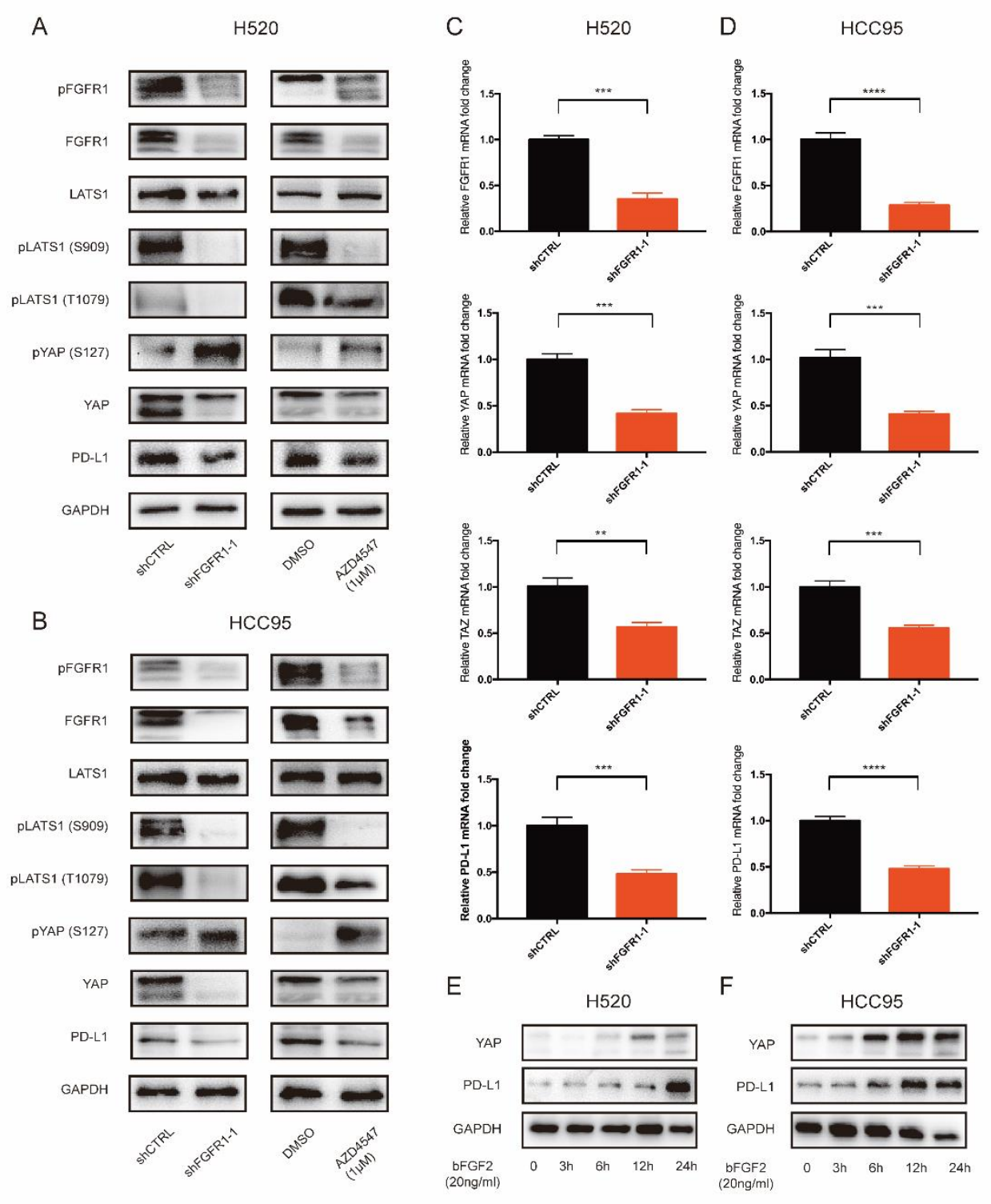

2 Figure 1. FGFR1 positively regulated YAP and PD-L1 expression at both the 3 mRNA and protein levels. (A-D) shFGFR1-1 or AZD4547 was added to the H520 and 4 HCC95 cell lines. (A, B) The levels of proteins related to the FGFR1/YAP/PD-L1 5 pathway were measured. $(C, D)$ The levels of mRNAs related to the FGFR1/YAP/PD6 L1 pathway were measured. $\mathrm{n}=3$, mean \pm SEM, two-tailed Student's t-test $(* \mathrm{p}<0.05$, $7 \quad * * \mathrm{p}<0.01, * * * \mathrm{p}<0.001, * * * * \mathrm{p}<0.0001$ ). (E, F) After activation of FGFR1 by 20 $8 \mathrm{ng} / \mathrm{ml} \mathrm{bFGF} 2$, the protein expression of YAP and PD-L1 was measured. 
Fig. 2

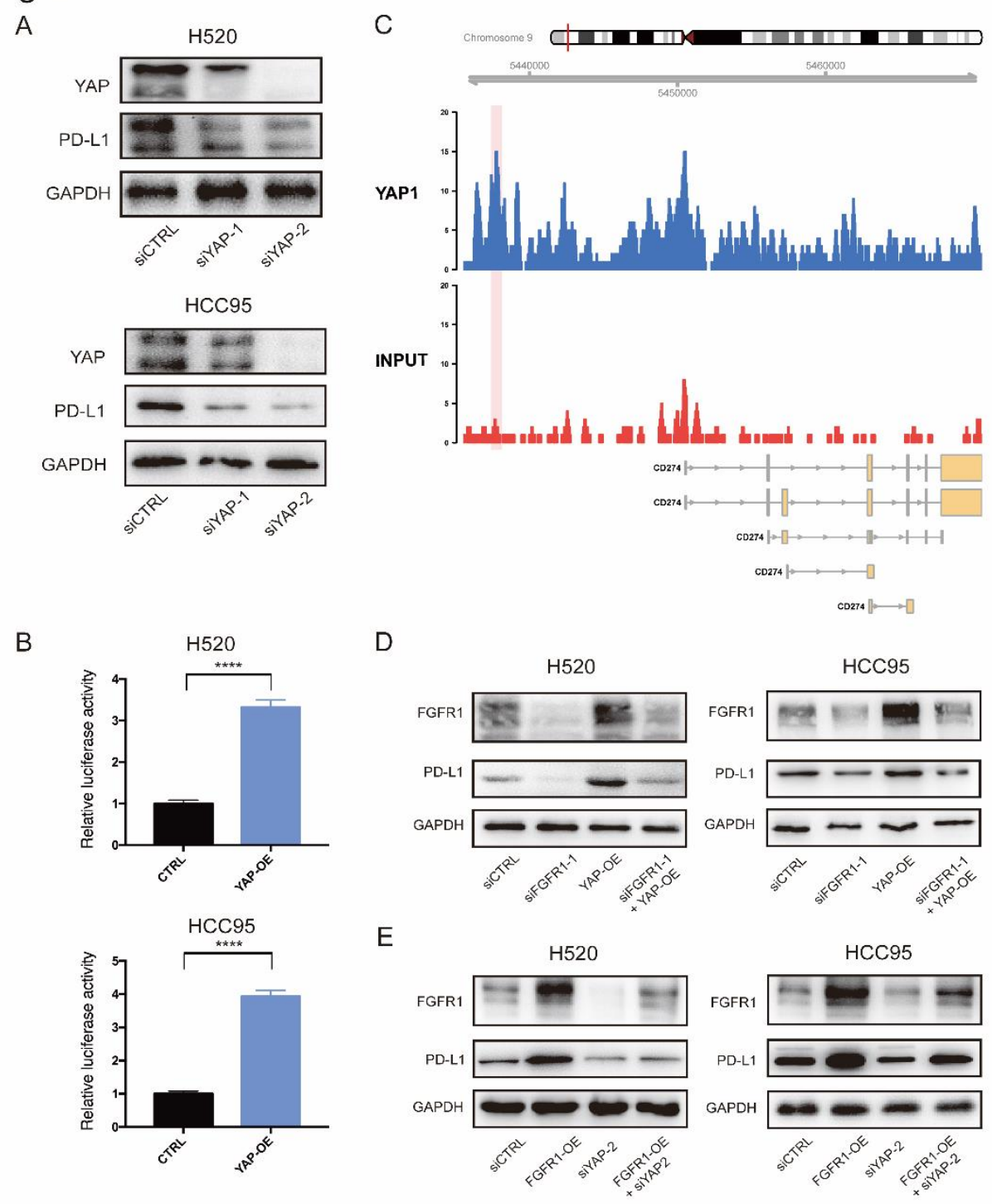

Figure 2. FGFR1 promoted PD-L1 expression through YAP, which directly bound to the promoter region of PD-L1. (A) In the H520 and HCC95 cell lines, siYAP sequence 1 and sequence 2 (siCTRL was used as a control) were added to inhibit YAP, and Western blotting was used to measure the protein expression of YAP and PD-L1. Sequence 2 was used subsequently. (B) After transduction of the YAP-CTRL or YAPOE (YAP overexpression) plasmid followed by the fluorescence-tagged PD-L1 promoter plasmid, relative fluorescence activity was determined $\left({ }^{*} \mathrm{p}<0.05,{ }^{*} \mathrm{p}<0.01\right.$, $* * * \mathrm{p}<0.001, * * * * \mathrm{p}<0.0001)$. (C) Chromatin immunoprecipitation (ChIP) revealed the interaction of YAP with a potential enhancer located upstream of the transcription starting site of PD-L1 on chromosome 9. Lysates were prepared from H520 cells and immunoprecipitated with IgG or 3-Flagged antibody. (D) siCTRL, siFGFR1-1, YAPOE and siFGFR1 plus YAP-OE siRNA or plasmid were added. (E) siCTRL, FGFR1OE (FGFR1 overexpression), siYAP-2 and FGFR1-OE plus siYAP-2 siRNA or plasmid were added. 
Fig. 3

A

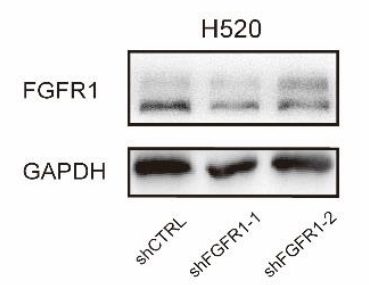

B

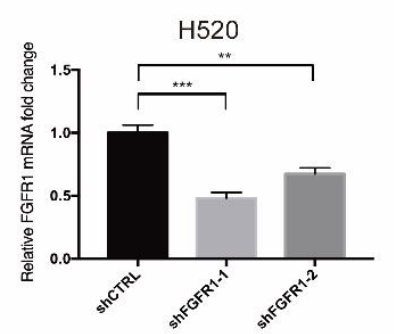

C

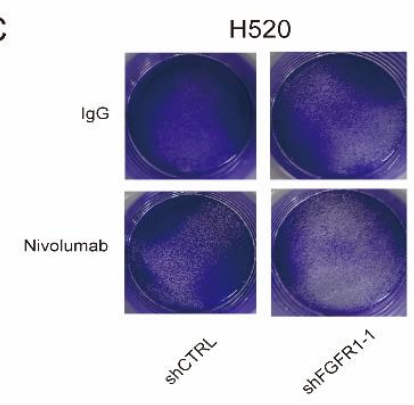

D

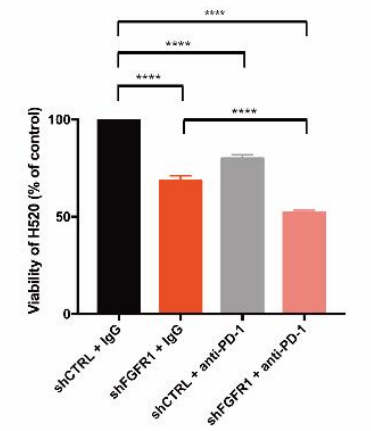

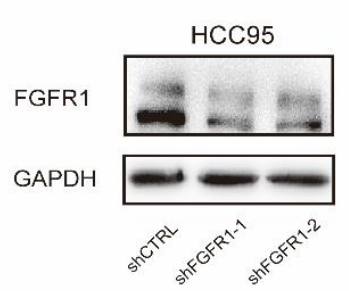
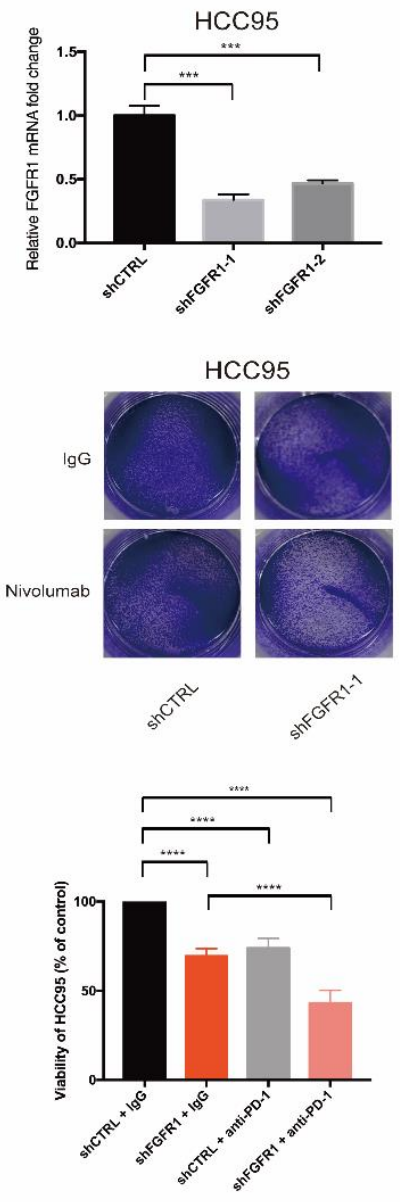

E Jurkat T cells
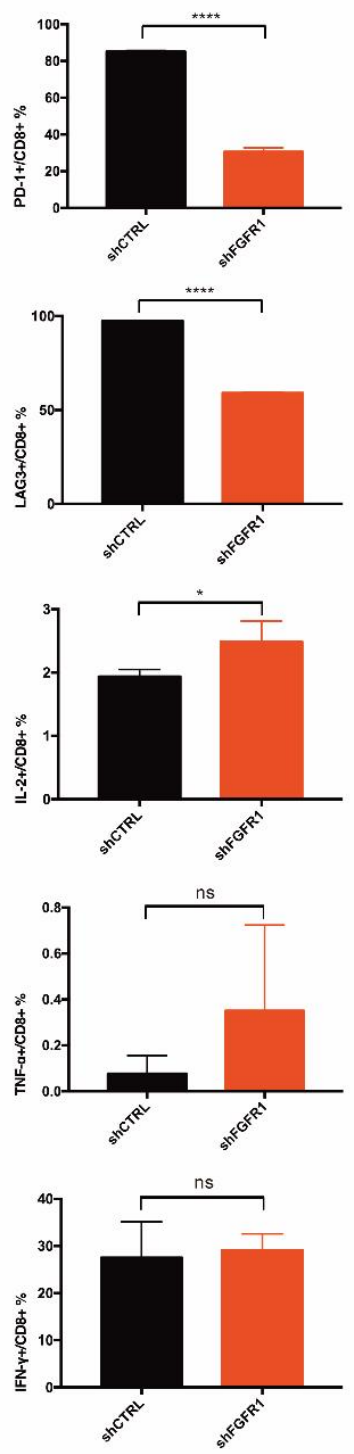

Figure 3. Knockdown of FGFR1 reversed immune evasion in vitro. (A) Western blot analysis of knockdown mediated by an shRNA-expressing lentivirus targeting FGFR1 (shFGFR1) in the H520 and HCC95 cell lines compared with the effect of the negative control lentivirus (shCTRL). (B) Confirmation of the knockdown mediated by shFGFR1 compared to the effect of shCTRL in H520 and HCC95 cells by qPCR. $\mathrm{n}=$ 3, mean \pm SEM, two-tailed Student's t-test. (C, D) H520 and HCC95 cells transfected with shCTRL or shFGFR1 were cocultured with Jurkat $\mathrm{T}$ cells and then treated with isotype IgG or nivolumab (a human anti-PD-1 mAb). Suspended T cells were removed, and adherent cancer cells were stained with crystal violet (C) or evaluated with the CCK-8 assay (D). The experiment was repeated twice. (E) Jurkat T cells were collected and tested by flow cytometry. $\mathrm{n}=4$, mean \pm SEM, two-tailed Student's t-test. $\left({ }^{*} \mathrm{p}<\right.$ $0.05, * * \mathrm{p}<0.01, * * * \mathrm{p}<0.001, * * * * \mathrm{p}<0.0001)$. 
Fig. 4
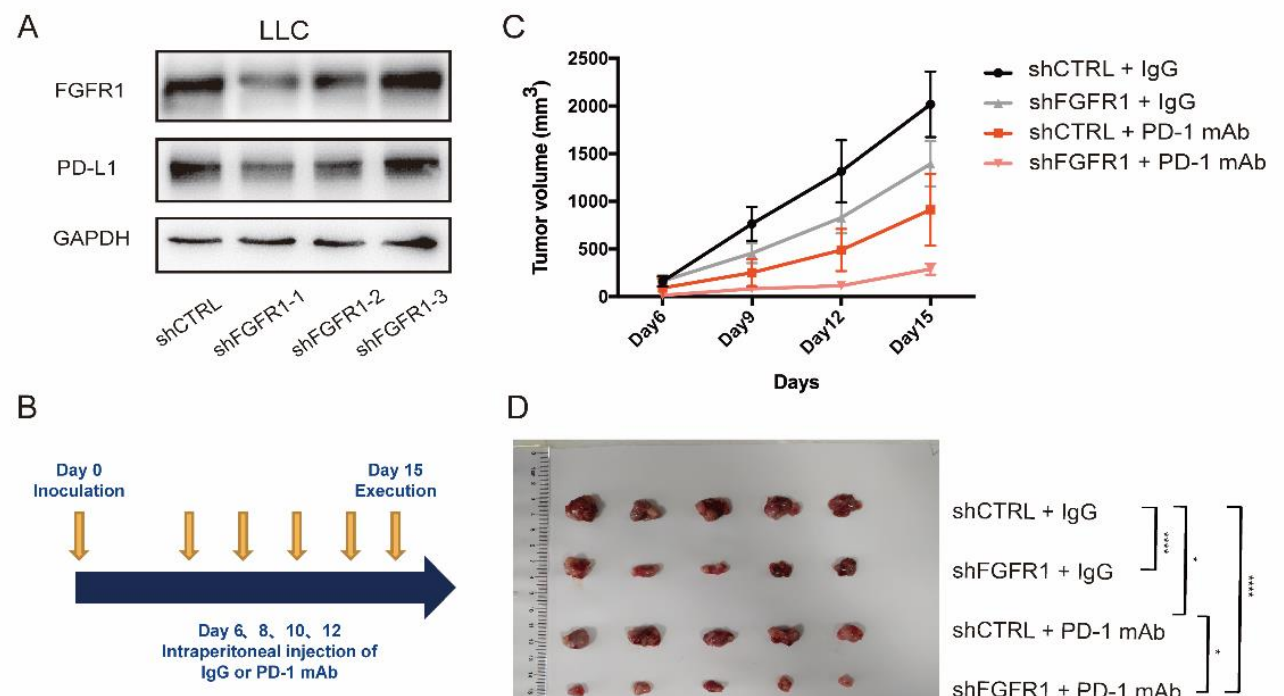

。
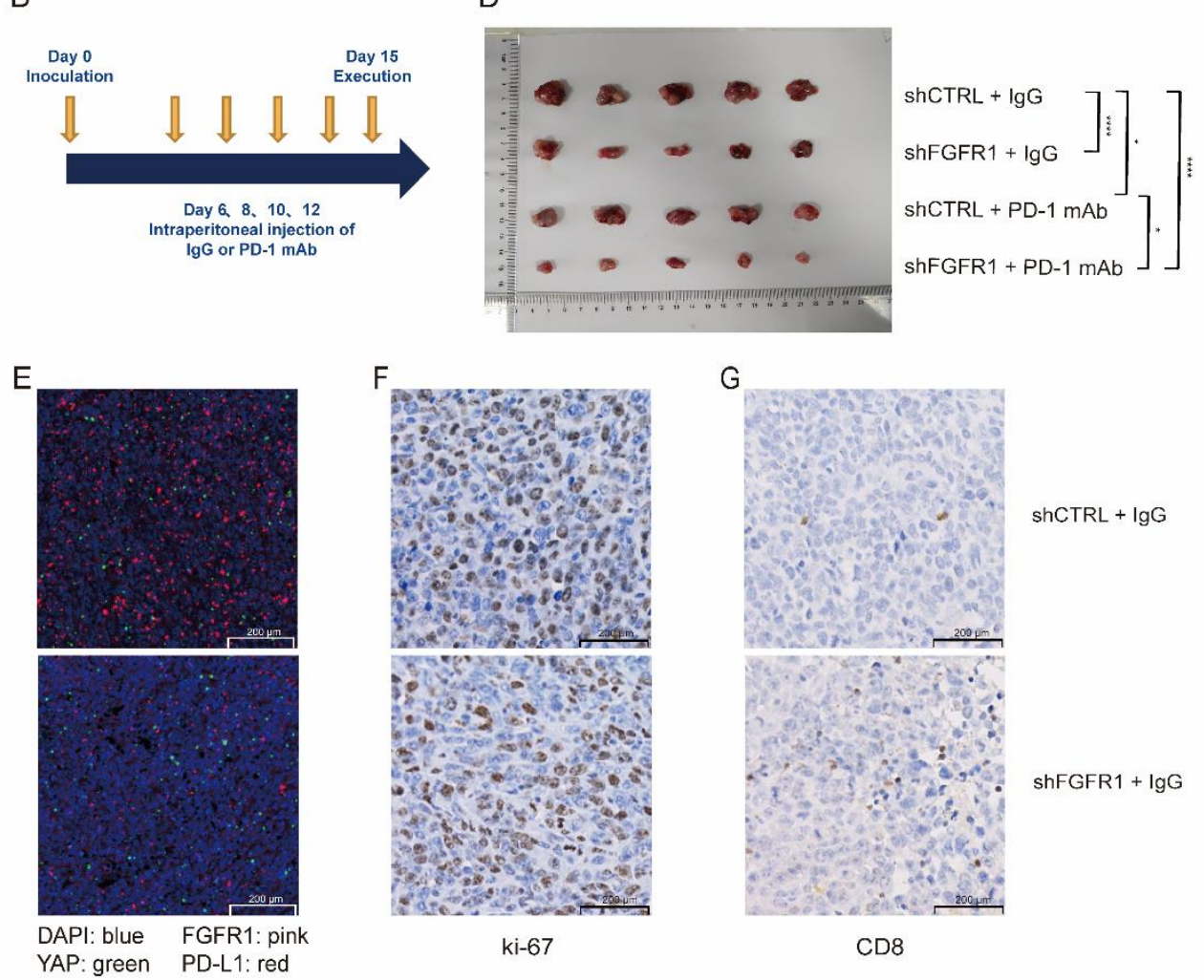

Figure 4. FGFR1 knockdown inhibited the FGFR1/YAP/PD-L1 axis and reduced tumor growth, especially in the presence of PD-1 antibodies. (A) Stable LLC mouse lung cancer cell lines were constructed by transduction of shFGFR1 sequences 1, 2 and 3 (shCTRL as a control). Western blotting was used to measures the protein expression levels of FGFR1 and PD-L1. shFGFR1 sequence 1 was subsequently amplified. (B) Schematic diagram of tumor inoculation and drug delivery. (C, D) Tumor photographs and growth curves of the subcutaneous tumor model in the shCTRL+IgG, shFGFR1+IgG, shCTRL+anti-PD-1, and shFGFR1+anti-PD-1 groups. $\mathrm{n}=5$, mean \pm SEM, one-way ANOVA followed by Bonferroni's multiple comparisons test $\left({ }^{*} \mathrm{p}<0.05\right.$, $* * \mathrm{p}<0.01, * * * \mathrm{p}<0.001, * * * * \mathrm{p}<0.0001$ ). (E) Multiple immunofluorescence (MIF) staining for DAPI (blue), FGFR1 (pink), YAP (green) and PD-L1 (red) in tumor tissues from the shCTRL+IgG and shFGFR1+IgG groups. (F, G) Analysis of tumor tissues in the shCTRL+IgG and shFGFR1+IgG groups by immunohistochemistry (IHC). (F) IHC for ki-67. (G) IHC for CD8. 
Fig. 5
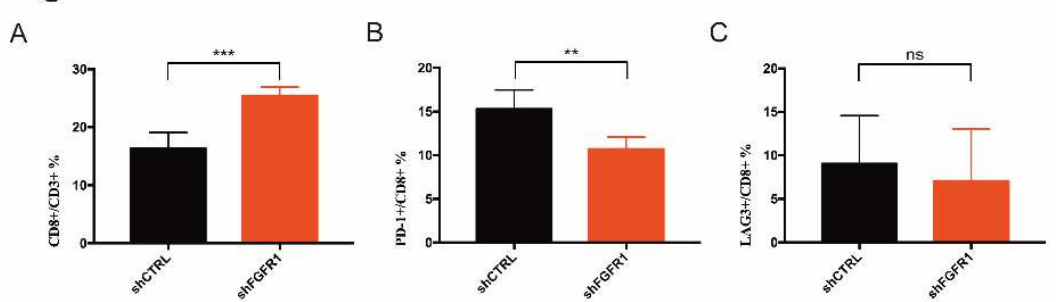

D

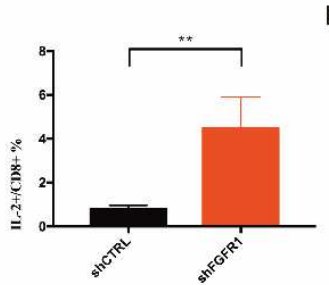

E
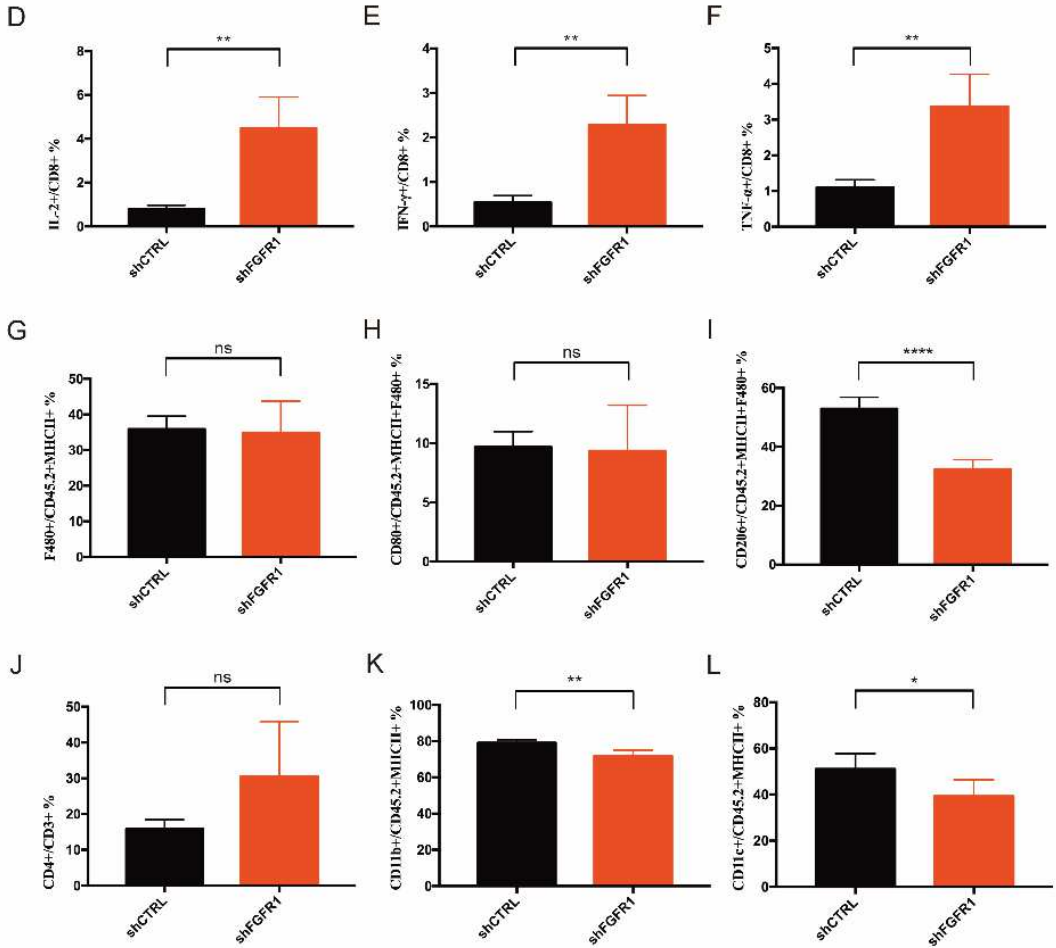

Figure 5. FGFR1 regulated CD8+ $T$ cells and additional immune cells in the tumor microenvironment. (A-L) Subcutaneous tumors were processed as single-cell suspensions and analyzed by flow cytometry. $\mathrm{n}=5$, mean \pm SEM, one-way ANOVA followed by Tukey's test $\left({ }^{*} \mathrm{p}<0.05,{ }^{*} \mathrm{p}<0.01, * * * \mathrm{p}<0.001, * * * * \mathrm{p}<0.0001\right)$. (A-F) Characteristics of CD8 $+\mathrm{T}$ cells are shown. (A) The percentage of CD8+ cells among the CD3+ cell population. (B) The percentage of PD-1+ cells among the CD8+ cell population. (C) The percentage of LAG3+ cells among the CD8+ cell population. (D) The percentage of IL-2+ cells among the CD8+ cell population. (E) The percentage of IFN- $\gamma+$ cells among the CD8+ cell population. (F) The percentage of TNF- $\alpha+$ cells among the CD8+ cell population. (G-I) Characteristics of macrophages. (G) The percentage of $\mathrm{F} 480+$ cells among the CD45.2+MHCII+ cell population. $(\mathrm{H})$ The percentage of $\mathrm{CD} 80+$ cells among the CD45.2+MHCII+F480+ cell population. (I) The percentage of CD206+ cells among the CD45.2+MHCII+F480+ cell population. (J) The percentage of CD4+ cells among the CD3+ cell population. $(\mathrm{K}, \mathrm{L})$ Characteristics of monocytes. $(\mathrm{K})$ The percentage of CD11b+ cells among the CD45.2+MHCII+ cell population. (L) The percentage of CD11c + cells among the CD45.2+MHCII+ cell population. 
Fig. 6
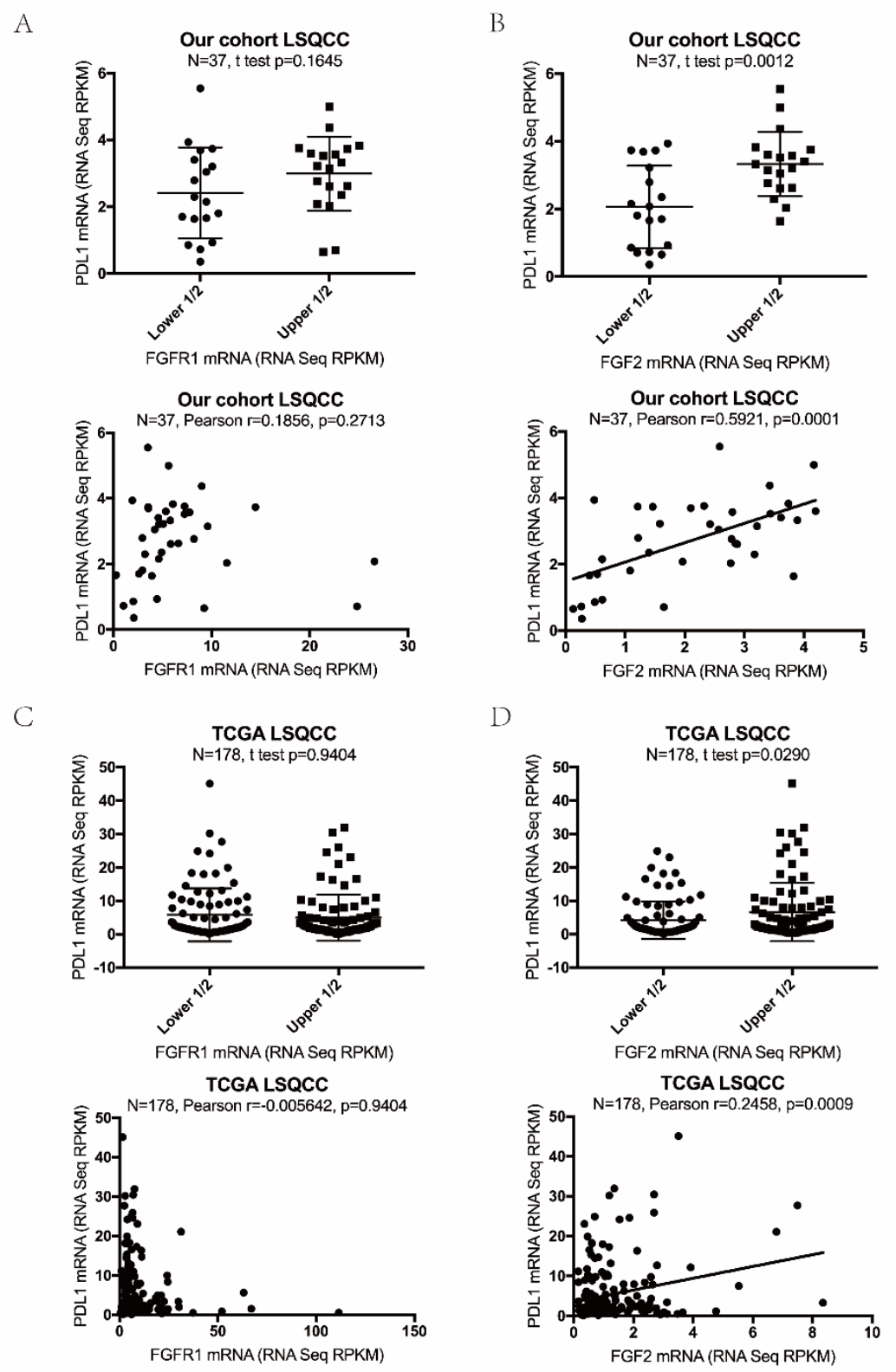

2 Figure 6. FGFR1 expression was correlated with PD-L1 expression and FGFR1

3 was coexpressed with PD-L1 in LSQCC patients from our institution. (A-D)

4 Linear regression analysis and Pearson correlation of the mRNA levels of FGFR1 and

5 PD-L1. (A, C) No statistical correlation between the expression of FGFR1 and PD-L1

6 was observed in either our cohort or the selected cohort of TCGA LSQCC patients.

7 (B, D) A positive correlation between the expression of FGF2 and PD-L1 was

8 observed in both our cohort and the selected cohort from TCGA LSQCC patients. 
Fig. 7

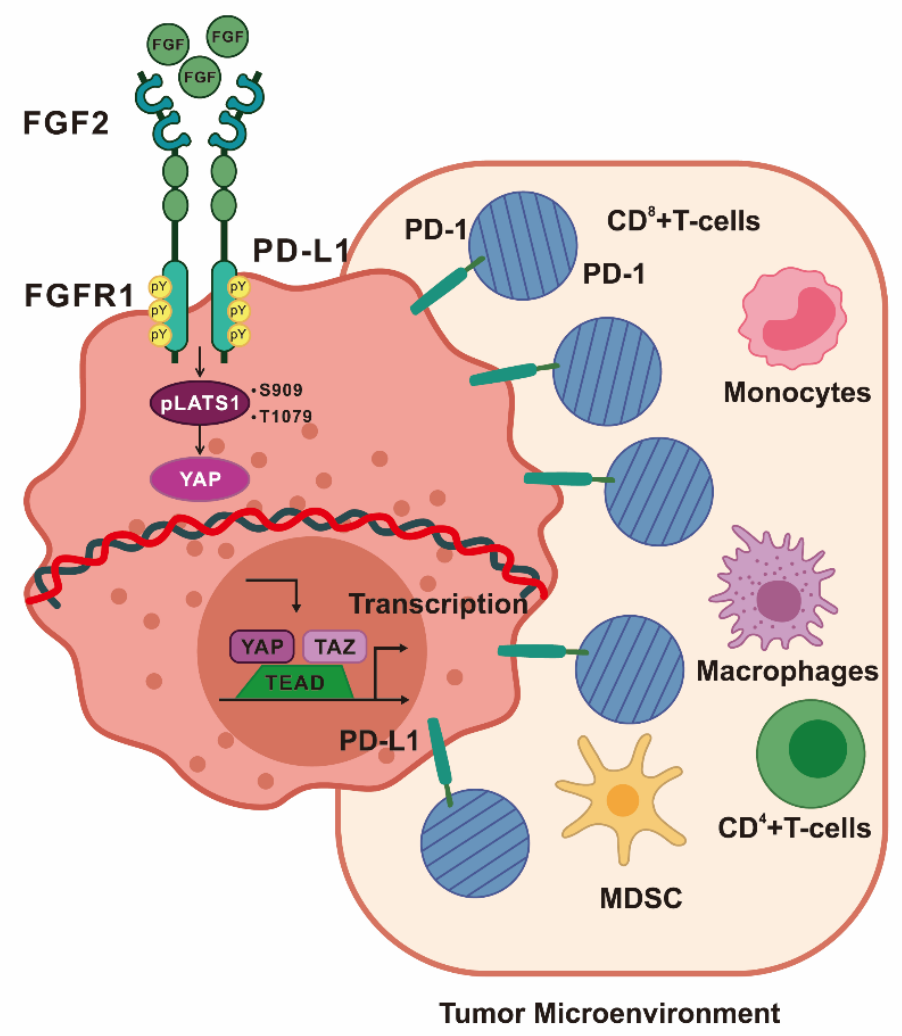

2 Figure 7. Schematic illustration of FGFR1's role in tumor immune evasion. 3

4

5

6 


\section{Supplementary Figure 1}

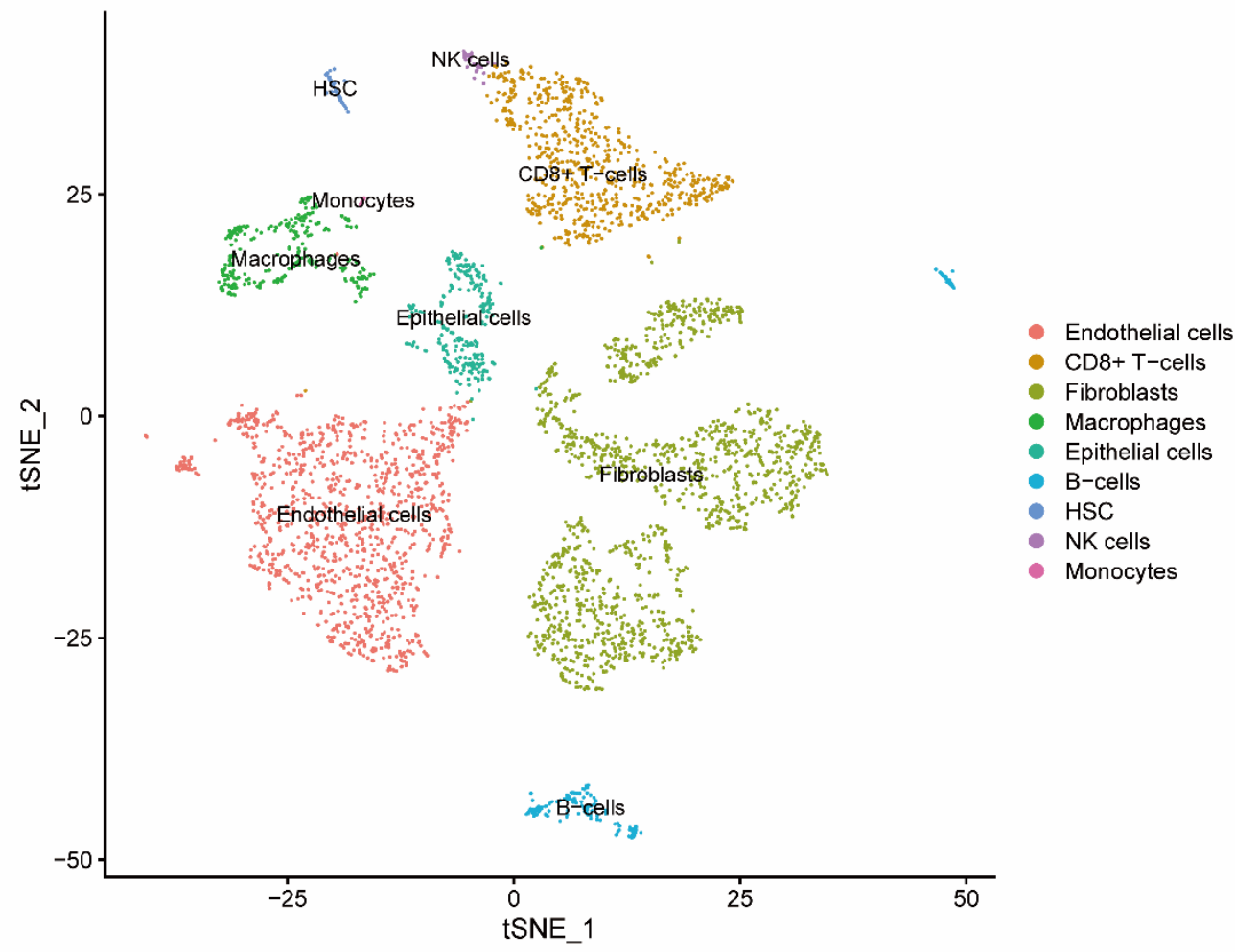

2 Supplementary Figure S1. Cell type composition in the tumor

3 microenvironment. Proportion of cells assigned to each cell type signature (color) 4 was visualized by tSNE. Endothelial cells, CD8+ T cells and fibroblasts were most 5 enriched as shown. Macrophages, epithelial cells, B cells, hematopoietic stem cells 6 (HSCs), natural killer cells (NK cells), and monocytes were also profiled. 
Supplementary Figure 2

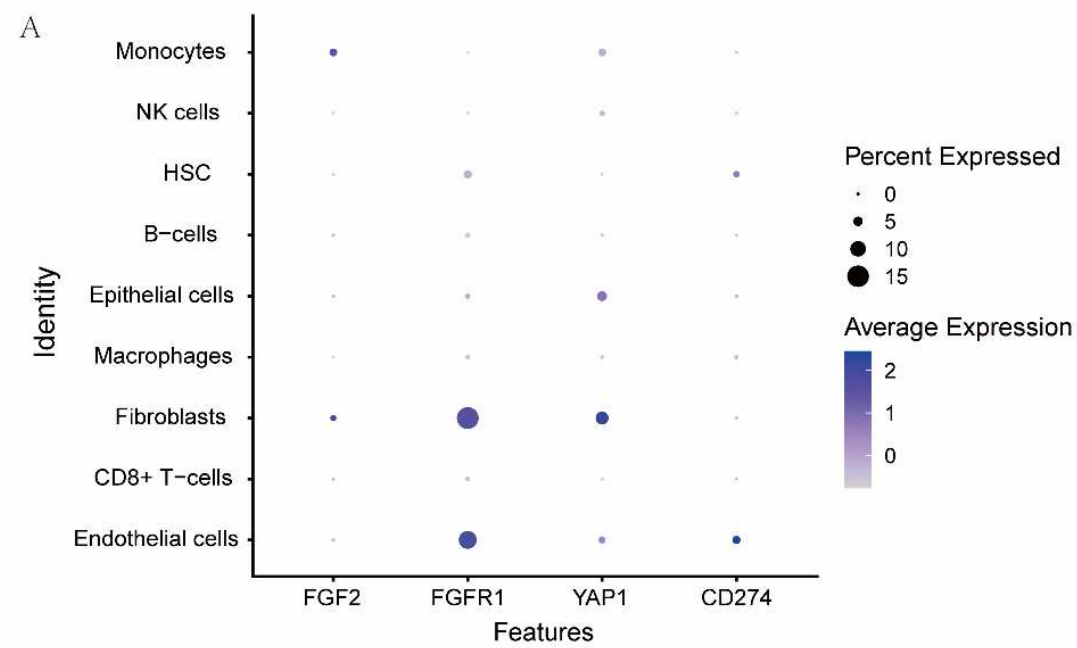

B
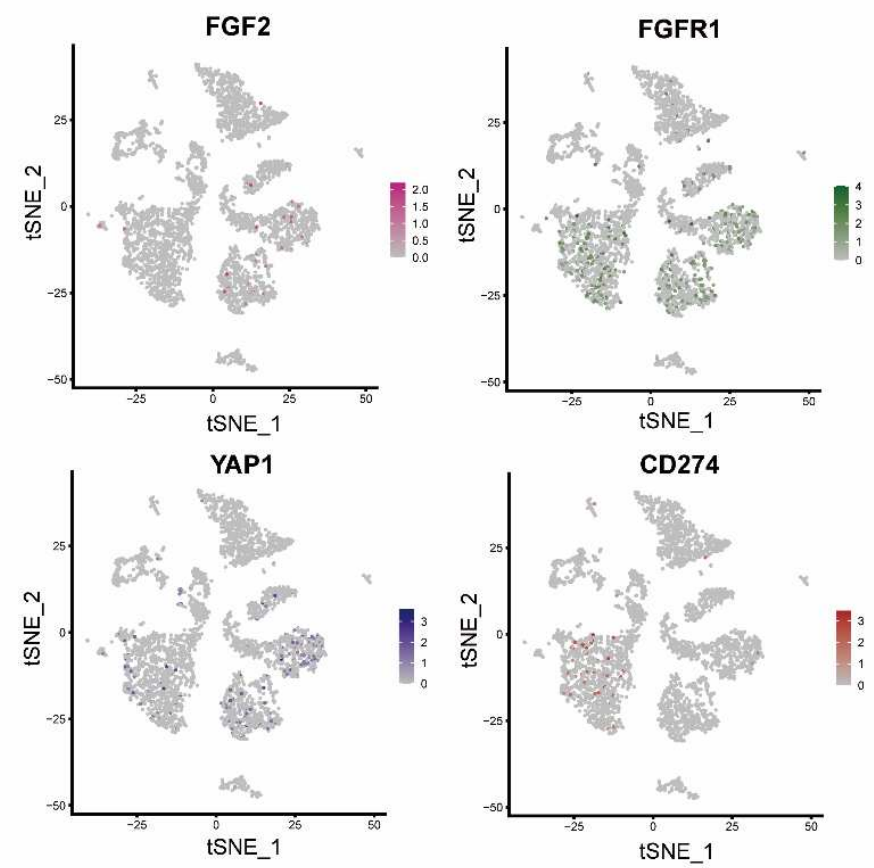

2 Supplementary Figure S2. FGF2, FGFR1, YAP and PD-L1 were coexpressed in

3 the TME. (A, B) Analysis of public single-cell sequencing data of LSQCC showed that FGF2, FGFR1, YAP and PD-L1 were co-expressed mainly in epithelial cells and fibroblasts in TME. (A) Bubble chart marked "FGF2", "FGFR1", "YAP1" (YAP) and "CD274" (PD-L1) in different PCs. The bubble size was proportional to the percentage of cells in the cluster that expresses the gene. The color intensity was proportional to the average scaling of the gene in the cluster. (B) Dimensionality reduction visualization map, highlighting "FGF2", "FGFR1", "YAP1", and "CD274" expression in "mediumvioletred", "darkgreen", "navyblue" and "firebrick" dots, respectively. 